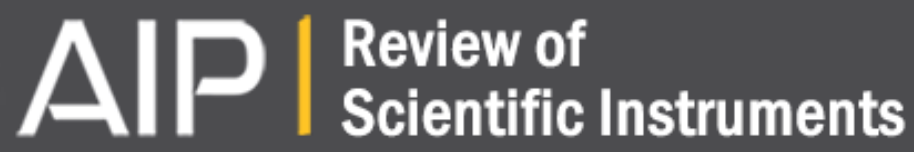

\section{Four-probe electrical measurements with a liquid pressure medium in a diamond anvil cell}

R. Jaramillo, Yejun Feng, and T. F. Rosenbaum

Citation: Review of Scientific Instruments 83, 103902 (2012); doi: 10.1063/1.4757178

View online: http://dx.doi.org/10.1063/1.4757178

View Table of Contents: http://scitation.aip.org/content/aip/journal/rsi/83/10?ver=pdfcov

Published by the AIP Publishing

\section{Articles you may be interested in}

Note: Achieving quasi-hydrostatic conditions in large-volume toroidal anvils for neutron scattering to pressures of up to $18 \mathrm{GPa}$

Rev. Sci. Instrum. 82, 076101 (2011); 10.1063/1.3606643

Finite element analysis of the effect of electrodes placement on accurate resistivity measurement in a diamond anvil cell with van der Pauw technique

J. Appl. Phys. 107, 104903 (2010); 10.1063/1.3374466

Finite element analysis of resistivity measurement with four point probe in a diamond anvil cell

J. Appl. Phys. 101, 064904 (2007); 10.1063/1.2712173

Technique for $\mathrm{x}$-ray markers at high pressure in the diamond anvil cell

Rev. Sci. Instrum. 76, 036102 (2005); 10.1063/1.1852327

Transport measurements at low temperatures in a diamond anvil cell with helium as pressure medium

Rev. Sci. Instrum. 68, 1514 (1997); 10.1063/1.1147639

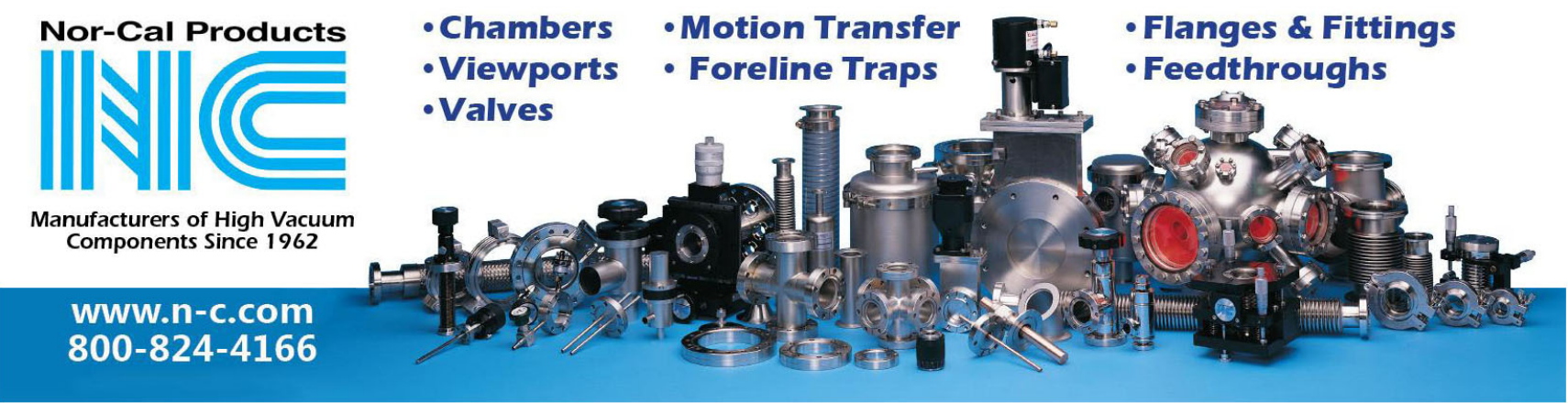




\title{
Four-probe electrical measurements with a liquid pressure medium in a diamond anvil cell
}

\author{
R. Jaramillo, ${ }^{1}$ Yejun Feng, ${ }^{2,3}$ and T. F. Rosenbaum ${ }^{3}$ \\ ${ }^{1}$ School of Engineering and Applied Sciences, Harvard University, Cambridge, Massachusetts 02138, USA \\ ${ }^{2}$ The Advanced Photon Source, Argonne National Laboratory, Argonne, Illinois 60439, USA \\ ${ }^{3}$ The James Franck Institute and Department of Physics, The University of Chicago, Chicago, \\ Illinois 60637, USA
}

(Received 2 August 2012; accepted 17 September 2012; published online 8 October 2012)

\begin{abstract}
We describe a technique for making electrical transport measurements in a diamond anvil cell using an alcohol pressure medium, permitting acute sensitivity while preserving sample fidelity. The sample is suspended in the liquid medium by four gold leads that are electrically isolated by a composite gasket made of stainless steel and an alumina-loaded epoxy. We demonstrate the technique with four-probe resistivity measurements of chromium single crystals at temperatures down to $4 \mathrm{~K}$ and pressures above $10 \mathrm{GPa}$. Our assembly is optimized for making high precision measurements of the magnetic phase diagram and quantum critical regime of chromium, which require repeated temperature sweeps and fine pressure steps while maintaining high sample quality. The high sample quality enabled by the quasi-hydrostatic pressure medium is evidenced by the residual resistivity below $0.1 \mu \Omega \mathrm{cm}$ and the relative resistivity ratio $\rho(120 \mathrm{~K}) / \rho(5 \mathrm{~K})=15.9$ at $11.4 \mathrm{GPa}$. By studying the quality of Cr's antiferromagnetic transition over a range of pressures, we show that the pressure inhomogeneity experienced by the sample is always below $5 \%$. Finally, we solve for the Debye temperature of $\mathrm{Cr}$ up to $11.4 \mathrm{GPa}$ using the Bloch-Gruneisen formula and find it to be independent of pressure. (C) 2012 American Institute of Physics. [http://dx.doi.org/10.1063/1.4757178]
\end{abstract}

\section{INTRODUCTION}

Using high pressure to continuously vary the volume of a sample is a conceptually direct way to test fundamental theories of solids, and to discover new and potentially important electronic and magnetic phases of matter. A good number of these discoveries have resulted from electrical transport studies of solids, which have become a mainstay of condensed matter physics and materials science because they provide direct insights into both single electron behavior and collective electronic effects. Hence, the ability to perform high quality electrical transport measurements at high pressures and low temperatures becomes an essential requirement for effectively probing new ground states and excitation spectra.

Cylinder-piston pressure cells are widely used for electrical measurements, but are limited to pressures below approximately $2.5 \mathrm{GPa}$, and electrical transport measurements at higher pressure remain a challenge. Furthermore, as with any extreme sample environment, measurement quality deserves elevated scrutiny. The purpose of this article is to describe in detail a technique that we used to make four-probe measurements of the resistivity and Hall coefficient of chromium in the vicinity of its antiferromagnetic quantum critical point near $10 \mathrm{GPa}$ and at cryogenic temperatures. ${ }^{1}$ Our technique is inspired by similar approaches reported elsewhere, to which we add adaptations that allow us to use a liquid alcohol pressure medium, essential to preserving sample quality for high resolution measurements. It is our hope that by describing our methods in great detail, we can encourage further experiments on phase transitions and fundamental properties of solids at high pressure.
We start by reviewing the options available for measurements of electronic transport at high pressure. First, there are several possible choices for the pressure vessel. Electrical measurements have been performed in a multi-anvil press, ${ }^{2}$ opposed-anvil pressure vessels such as toroid cells, ${ }^{3}$ Bridgman cells, ${ }^{4-7}$ and diamond anvil cells (DAC). ${ }^{1,8-17}$ The latter two are often the most convenient choices for measurements at cryogenic temperatures due to their limited mass and size. For measurements of critical phenomena, for which fine tuning and precise measurement of the pressure are of the utmost importance, diamond anvil cells are a suitable choice. With a helium-gas membrane providing the driving force, the sample pressure can be reliably adjusted in increments of less than $0.1 \mathrm{GPa}$, and in situ measurement using the ruby fluorescence technique can provide precision below $0.01 \mathrm{GPa}$. Therefore, we focus our discussion on electrical transport techniques for diamond anvil cells.

We have noted previously that in order to minimize pressure inhomogeneity and anisotropy experienced by the sample, it is important that the ratio of pressure chamber volume to sample volume be as large as possible. ${ }^{18}$ In addition to our work with diamond anvil cells, this idea is supported by high quality measurements in Bridgman cells, ${ }^{4-6}$ in which a relatively large pressure chamber is available. It is also desirable to start with a soft pressure medium, that is, in the liquid form upon loading, ${ }^{2,4-6,17}$ especially for diamond anvil cells in which the pressure chamber volume is especially constrained.

Most electrical transport measurements at high pressure reported to date, either with diamond anvil or other types of pressure cell, have used a solid pressure medium such 
as steatite, ${ }^{7} \mathrm{AgCl},{ }^{19} \mathrm{NaCl},{ }^{14}$ or cubic-BN-epoxy mixture. ${ }^{13}$ Sometimes, with samples such as lithium and europium that are especially soft and reactive, no pressure medium was used. $^{20,21}$ Several groups have demonstrated so-called designer diamond anvils that have electrical leads lithographically patterned on the diamond culet. ${ }^{22-24}$ In these cases, it appears necessary to use either a solid pressure medium or no pressure medium in order to ensure reliable contact between the sample and the leads. For experiments using a solid pressure medium, the electrical leads are often pressed against the sample by the anvil to make contact. This procedure can be particularly damaging for single crystal samples, which should not be mechanically deformed. For all these reasons, a liquid pressure medium with securely attached electrical leads is preferred for maintaining sample quality throughout the high-pressure experiment.

\section{EXPERIMENTAL ASSEMBLY: COMPOSITE GASKET FOR A LIQUID PRESSURE MEDIUM}

Our experimental method is designed for high-resolution studies such as phase transitions and critical phenomena in solids at low temperature and at pressures up to approximately $12 \mathrm{GPa}$. In this regime, a 4:1 methanol:ethanol pressure medium is known to produce quasi-hydrostatic conditions, and the most important design criterion for maintaining high sample quality is that the sample volume is small relative to the pressure chamber. ${ }^{18}$ We therefore opt for large diamond anvils and a correspondingly large pressure chamber. The anvils are type Ia, 16-sided, Drukker design (Almax Industries, Belgium). Our typical assembly uses a bottom anvil with a $1 \mathrm{~mm}$ diameter flat culet, a top anvil with a $0.83 \mathrm{~mm}$ culet and an $8^{\circ}$ bevel out to $1 \mathrm{~mm}$. According to Ref. 25, the theoretical high-pressure limit for a diamond anvil with a $1 \mathrm{~mm}$ culet is approximately $12.5 \mathrm{GPa}$. The pressure chamber is $450 \mu \mathrm{m}$ diameter $\times 90 \mu \mathrm{m}$ height, which is large enough to comfortably contain our $(200 \times 200 \times 40) \mu \mathrm{m}^{3}$ sample with four electrical leads.

The principal challenge in making electrical transport measurements in a diamond anvil cell lies in introducing electrical leads into the pressure chamber while avoiding electrical contact to the metallic gasket. Some groups avoid this problem by using a gasket made entirely from an electrical insulator such as mica, ${ }^{8}$ fiber-diamond powder-Stycast mixture, ${ }^{17}$ or a mixture of epoxy and cubic boron nitride powder. However, the mechanical advantages of metal gaskets are significant, and most researchers prefer to use a metal gasket and apply an insulating layer to isolate the electrical leads. For this work, we also prefer a composite gasket with both metal and ceramic layers to better contain the liquid pressure medium and minimize both the sample chamber deformation and disintegration of the electrical insulation layer. Similar to other insulating coatings reported in literature, ${ }^{8-13,15,16}$ we combine $\mathrm{Al}_{2} \mathrm{O}_{3}$ powder and epoxy in a mixture ("ceramic epoxy" below), that is, both electrically insulating and compatible with a liquid alcohol pressure medium.

We start the gasket preparation with a bare, fully hardened 301-type stainless steel shim stock of $250 \mu \mathrm{m}$ thickness, which is cut into a square of approximately $1 \mathrm{~cm}^{2}$. The metal

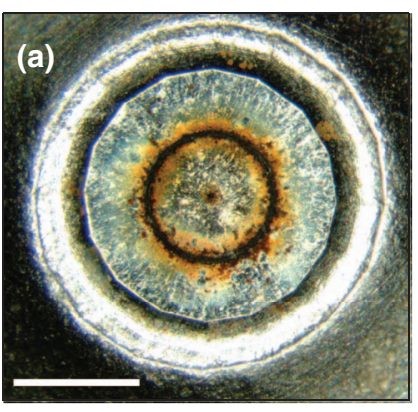

(b)
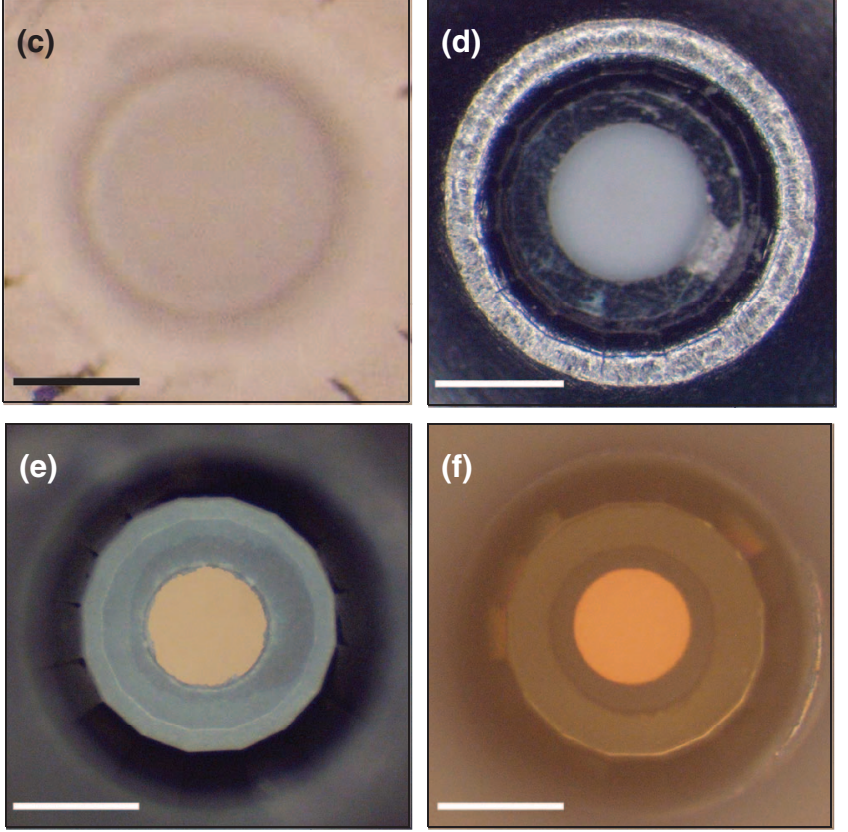

FIG. 1. Step-by-step gasket preparation. Scale bars are $500 \mu \mathrm{m}$. (a) Metal gasket after initial indentation and laser drilling. (b) Schematic of the indentation of a coated gasket after the ceramic epoxy has been drop cast and cured. The top epoxy surface is lumpy; the bottom side of the gasket is smooth with a clean metal-epoxy interface. (c) and (d) Top and bottom sides after indenting the coated gasket. (e) and (f) Top and bottom sides after drilling out the sample chamber

backbone of the composite gasket is prepared similarly to a normal DAC gasket: the shim is indented to approximately the same thickness that we intend for the eventual composite gasket, which in this case is in the range 90-100 $\mu \mathrm{m}$. We next drill a hole in the indented metal gasket, preferably using a non-mechanical technique such as laser drilling (Fig. 1(a)) or electrical discharge machining to produce clean edges and sidewalls. In our experience, mechanical drilling leaves burrs that interfere with the epoxy coating, and could short the electrical leads. Since the eventual pressure chamber walls will be coated in ceramic epoxy, the drill hole diameter at this stage should be larger than the intended chamber diameter. For our experiment, the optimal range for this drill hole diameter is $500 \pm 20 \mu \mathrm{m}$. For holes smaller than this range, we find that the ceramic epoxy layer that coats the pressure chamber walls is too thin, and is likely to leave spots of exposed metal. For holes larger than this range, we find that after pressing the composite gasket (metal + ceramic epoxy), the metal is often completely extruded from the culets, resulting in an allceramic gasket with a metal girdle. Such a design has been used successfully by others, ${ }^{8,16}$ but for this work we prefer 
a composite gasket with both metal and ceramic layers. In Fig. 1(a), we show a gasket which has been laser drilled. The laser perforates the metal, leaving a burn track, and the central disc is easily pushed away.

After being drilled, one side of the metal gasket is coated in ceramic epoxy. For our assembly, we found that the epoxy should satisfy several criteria. After being cast and cured it should still deform plastically, so that it can be pressed in the DAC to the desired shape. It should adhere to the metal gasket, but not stick to the diamond while being pressed into shape. It should have the right density and viscosity to be drop-cast to an appropriate thickness. It should be amenable to mechanical drilling without chipping, so that a clean sample chamber can be drilled out. It should not dissolve or disintegrate in the pressure medium. Finally, it should be electrically insulating and withstand the pressures and temperatures reached in the experiment. We find that these criteria are met by a mixture of $\mathrm{Al}_{2} \mathrm{O}_{3}$ powder (sub-micrometer particle size, Atlantic Equipment Engineers) and UV-curable epoxy (NOA81, Norland Products) in a 4:1 (powder:epoxy) mass ratio. This is a $58 \%$ volume ratio for alumina, which is close to the volume ratio $(64 \%)$ for dense random packing of monodisperse spheres. To mix the powder and epoxy, we use acetone as a solvent and stir. The viscosity of this mixture changes continuously as the solvent dissolves. As a result, the thickness of a layer formed by drop casting also changes continuously. This provides some control over the thickness of the epoxy layer, but also imposes a finite time window in which the epoxy should be drop cast onto the gasket. We find that an appropriate layer is formed by drop casting when the mass ratio is approximately 3:4:1 (acetone:powder:epoxy). We use a wooden stick to transfer one or two drops of epoxy from the mixture to the center of the gasket. If drop cast at the appropriate viscosity, then the epoxy will fill the gasket hole but will not cover the bottom side: surface tension will produce a clean metalepoxy interface. The gasket has to be supported so that the bottom side and edges are not in contact with other surfaces in order to avoid the epoxy being pulled by capillary action across the bottom surface of the gasket. For our experiment, with all electrical leads lying on one side of the gasket, it is only necessary to coat one side with epoxy and the metallic surface on the bottom side of the gasket should remain clean.

After drop casting, we cure the ceramic epoxy by placing the gasket under an ultraviolet lamp for up to one hour. The insulating layer that results is hard and uneven, with no surface to match the top anvil. In Fig. 1(b), we show a schematic of a gasket at this stage, with a lumpy epoxy layer coating the top side and a clean interface between epoxy and metal underneath. We now indent the composite gasket to the desired final thickness in the DAC. During this indentation both the epoxy and the metal will be compressed and extruded, and the epoxy-filled hole will expand. Since the epoxy is at first uneven, we often find that the diamonds become misaligned during this indentation. We therefore frequently remove the partially indented gasket and check the alignment of the anvils, making adjustments as necessary. For our experiment, we indent to a final thickness of $90-100 \mu \mathrm{m}$, by which point the ceramic epoxy is translucent and the metal hole is expanded to roughly $650 \mu \mathrm{m}$ in diameter. A fully indented composite gasket is shown from the top and the bottom in Figs. 1(c) and 1(d). The epoxy surface is now optically smooth.

We then drill out the sample chamber from the pressed epoxy using a high speed mechanical drill equipped with an inspection microscope. The compressed epoxy is not well sintered and is easily drilled with conventional drill bits; we use either bright finish (Minitool) or TiN-coated (Guhring) high speed steel bits. We drill this hole in multiple stages, starting with a 50 or $100 \mu \mathrm{m}$-diameter guiding hole and gradually stepping up to the desired $450 \mu \mathrm{m}$ diameter. At each stage, we take care to drill part-way from both the front and back instead of simply drilling through from one side. This multistep procedure is meant both to avoid chipping, and to allow for corrections to be made in case of a misalignment of the initial guiding hole. In Figs. 1(e) and 1(f), we show a gasket with the sample chamber finished.

\section{EXPERIMENTAL ASSEMBLY: SAMPLE AND SPOT-WELDED ELECTRICAL LEADS}

For samples to be successfully suspended in a liquid pressure medium, it is essential to establish robust mechanical and electrical contact between the sample and the leads. Given the uncertain temperature and pressure evolution of contact resistance, true four-probe measurements in either a long-bar or a van der Pauw geometry ${ }^{26}$ are necessary for high-fidelity results. Metallic leads are often attached by conductive paint/epoxy/paste made of graphite, ${ }^{17}$ gold, ${ }^{12}$ or silver ${ }^{9}$ sub-micron powders. This method is considered to be the least damaging for fragile samples such as lowdimensional and organic materials. However, we have observed that silver epoxy contacts are not stable when exposed to a methanol:ethanol 4:1 mixture. In such cases, a different liquid pressure medium is typically used, such as glycerol, ${ }^{9}$ Phomblin oil, ${ }^{17}$ fluorinert, ${ }^{12}$ or iso-n-pentane. An alternate method for making electrical contacts is spot welding. ${ }^{27,28}$ Spot welding typically provides a contact resistance several orders of magnitude smaller than conductive paste and therefore yields reduced electrical noise, which can be a determinative consideration for the success of the experiment when measuring highly conductive samples. Furthermore, spot welded contacts are compatible with any pressure medium. Spot welding requires highly conductive samples and a welder specialized for making microscopic contacts. In addition, care must be taken not to damage the sample through excessive heating.

For our experiment with highly conductive metal samples, we have developed a reproducible spot welding technique for attaching electrical leads that preserves crystal quality. We prepared single crystal chromium samples of size (200 $\times 200 \times 40) \mu \mathrm{m}^{3}$ from large crystal boules; details of this sample preparation have been reported elsewhere. ${ }^{29}$ We press the lead wires, made from $25 \mu \mathrm{m}$ gold wire, onto the sample surface using a tungsten blade. The sample is grounded, and an electrical pulse is applied through the blade using a home built miniature spot welding system. ${ }^{27,28}$ Typical pulse parameters for robust connections are $30 \mathrm{~V}$ and $5 \mu \mathrm{s}$. Under these conditions the damage to the single crystal sample is 

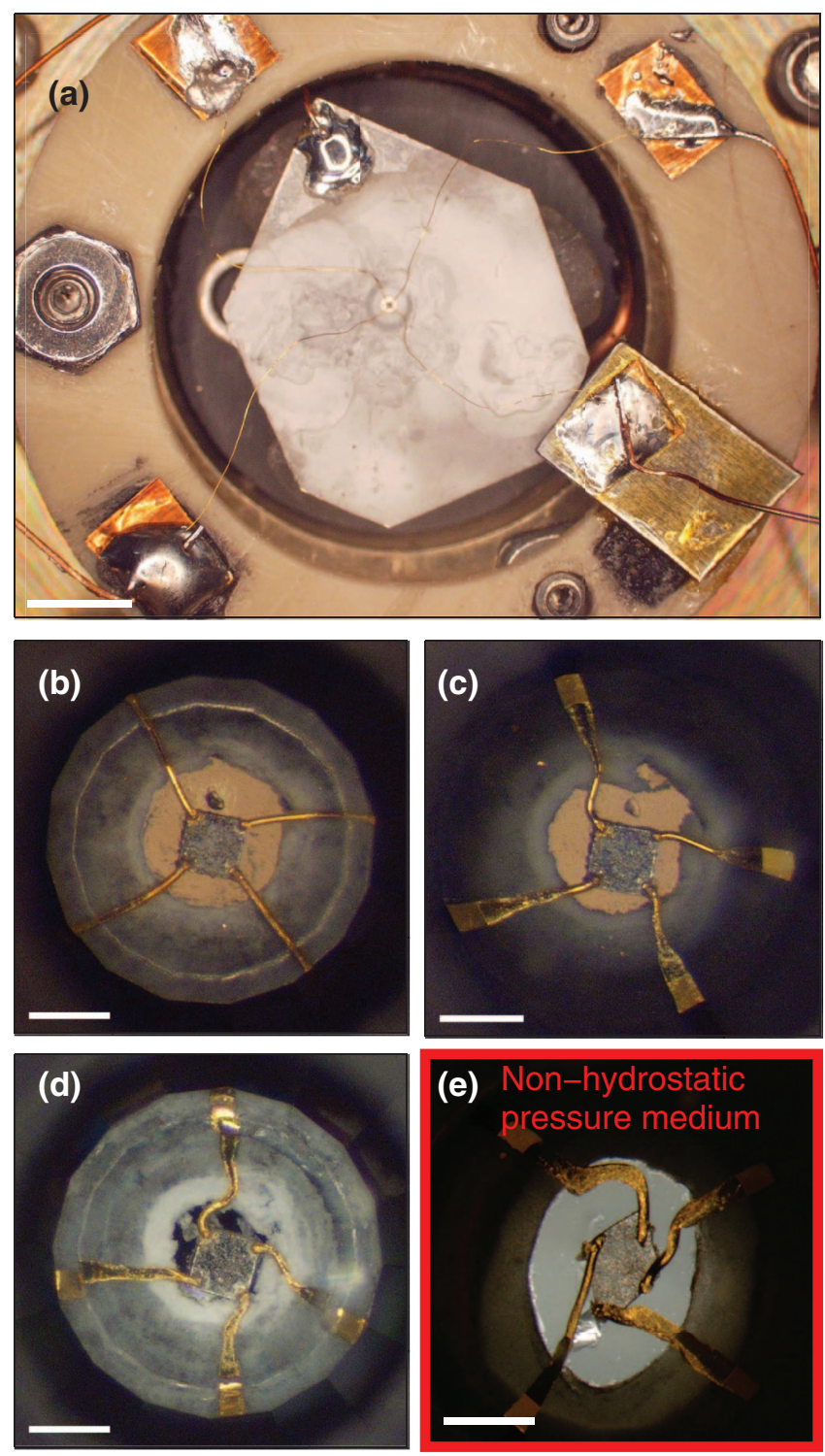

FIG. 2. Sample in the pressure chamber. Scale bar is $4 \mathrm{~mm}$ in (a) and $250 \mu \mathrm{m}$ in (b)-(d). Panels (a)-(d) show preparations for a single experiment in chronological order. (a) Assembly before placing the top anvil. (b) View through the top anvil before loading the cell, with the wires not yet flattened. A ruby chip used as a pressure gauge is visible above the sample. (c) After loading to an initial low pressure and before cooling down in the cryostat. (d) Image taken after the lower anvil ruptured at $11.5 \mathrm{GPa}$ at low temperature. (e) Image of another experiment, using silicone oil instead of methanol:ethanol as the pressure medium. The severe distortion of the sample results from non-hydrostatic conditions.

negligible, as can be seen visually (Figs. 2(b) and 2(d)) and through the measured residual resistivity (Fig. 3(a)).

After spot welding, the four leads are connected to breakout wires on a plastic supporting ring. The ring is mounted to the body of the cell with set screws, providing the requisite freedom of adjustment to position the sample in the pressure chamber. We show in Fig. 2(a) a micrograph of the assembly prior to adjusting the sample position. The sample is at the center; the four gold leads extend beyond the gasket to breakout pads on the plastic support ring. The gasket is almost fully coated with ceramic, with the exception of a soldered contact, which is used to monitor the electrical insulation of the
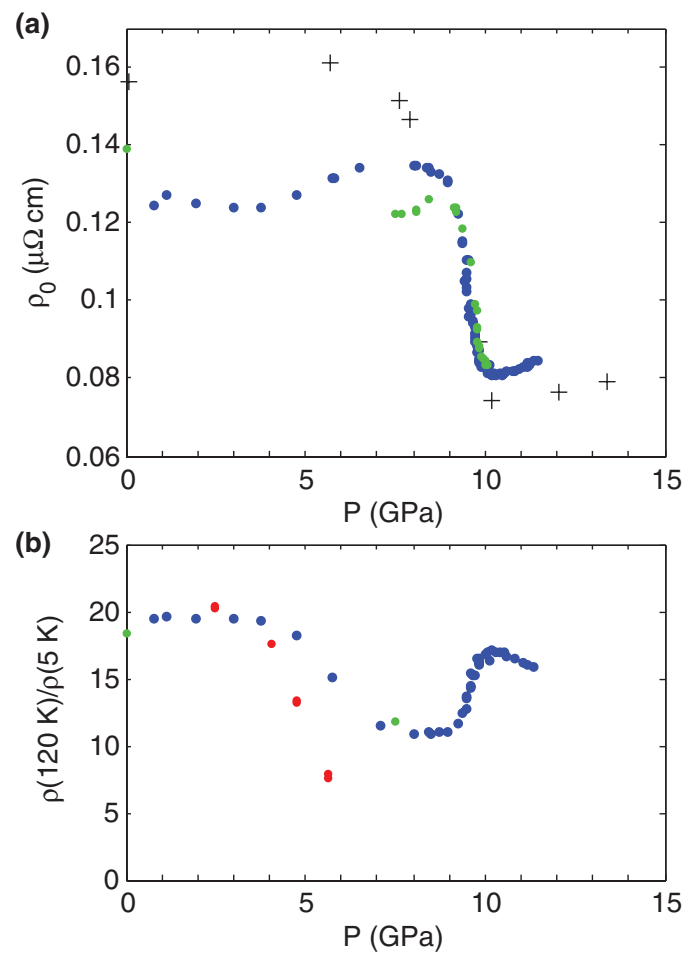

FIG. 3. Residual resistivity and relative resistivity ratio of chromium vs pressure. (a) $\rho_{0}$ vs pressure; blue and green circles were measured on two different samples using the techniques described here. Black crosses are reproduced from Ref. 7. (b) $R_{R R} 120$ vs pressure; symbols are the same as in panel (a). For experiments with an alcohol pressure medium (blue and green points) $\mathrm{RRR}_{120}$ remains relatively unchanged between ambient and high pressure, and largely reflects the antiferromagnetic phase diagram. With a silicone oil pressure medium (red points) $\mathrm{RRR}_{120}$ falls rapidly as the sample is damaged.

leads. We show in Fig. 2(b) a micrograph of a sample that has been positioned in the pressure chamber. This image is taken through the top anvil, which has been closed to the point of touching but not flattening the leads. The freestanding gold leads, which provide excellent electrical and mechanical contact to the sample, pose problems when attempting to keep the sample positioned in the chamber while the cell is loading. Meticulously positioning the leads on the gasket can be time consuming and frustrating, but is essential for a successful experiment. At this point, we introduce the alcohol pressure medium, using a syringe needle to flood the pressure chamber. We find that opening a small vertical gap between the gasket/sample/lead assembly and the upper anvil is helpful to avoid trapping air bubbles in the pressure chamber while loading.

The gold wires are pressed into ribbons by the top anvil, as shown in Fig. 2(c). Although it is conceivable that the wires could break at the points where they enter the pressure chamber or short to the underlying metal gasket, in practice, neither of these failure modes has occurred even at the highest measured pressures. The insulation between the leads and the gasket metal typically remains above $20 \mathrm{M} \Omega$ (copper wires on pressed alumina are reported to be similarly robust. ${ }^{11}$ ) Instead, the most frequent failure mode of the gold wires occurs during cell loading. Once the wires are pressed into ribbons they become delicate and difficult to reposition. If the wires are flattened but the sample is misplaced and needs to 
be repositioned, then doing so runs a high risk of breaking the ribbons.

We also see in Fig. 2(c) that a wisp of epoxy has detached from the gasket wall and appears to be floating in the pressure chamber, immediately after we sealed the cell at low pressure. This is not uncommon; upon first contact with the liquid pressure medium, loose pieces of epoxy will often detach from the gasket wall. This does not pose a problem for the experiment. Most importantly, the bulk of the epoxy gasket, which is supported by the large contact area with the metallic part of the gasket, remains well bound and does not dissolve or otherwise disintegrate with submersion in alcohol. We have verified this by repeated loading (to low pressure) and unloading of individual gaskets, with the pressure medium allowed to completely evaporate in between unloading and re-loading. After any loose pieces of epoxy are removed by initial contact with the liquid pressure medium, the gasket remains intact and viable.

Once the cell is successfully loaded, the leads are robust and they almost never break in or near the pressure chamber. In fact, even catastrophic failure due to an anvil rupturing at high pressure may not break the electrical connection to the sample. We show in Fig. 2(d) a micrograph taken after the lower diamond ruptured at $11.5 \mathrm{GPa}$. All spot welded contacts on the sample remain intact, although three of the four wires did break at the outer edge of the culet.

The effects of using a non-ideal pressure medium are vividly illustrated in Fig. 2(e). Here we show a micrograph of a sample in the pressure chamber after having been pressurized to above $5.5 \mathrm{GPa}$ at low temperature with silicone oil as the pressure medium. The originally square sample has been distorted into a parallelogram. The degradation of the sample quality is also evident through the resistivity data, see below. This does not imply that silicone oil is a poor choice for other high pressure vessels, but it appears to be a poor choice for DACs with limited sample chamber volume.

\section{RESULTS: CHROMIUM RESISTIVITY AT HIGH PRESSURE AND LOW TEMPERATURE}

Chromium is the archetypical spin density wave (SDW) antiferromagnet. Above the Néel temperature $\left(T_{\mathrm{N}}\right)$, it is paramagnetic and metallic. Below $T_{\mathrm{N}}$ a fraction of the Fermi surface becomes gapped by the SDW, producing the characteristic minimum in the resistivity at $T_{\mathrm{N}}$. Only a fraction of the paramagnetic Fermi surface participates in the SDW, and chromium remains a good metal down to the lowest temperatures. At ambient pressure $T_{\mathrm{N}}=311.5 \mathrm{~K}$, and the transition temperature can be continually suppressed to zero with applied pressure. ${ }^{1}$

We performed electrical resistivity and Hall coefficient measurements in a helium flow cryostat. Our home built pressure cell contains a helium membrane that we use to control the sample pressure via a gas capillary line installed in the cryostat. Our typical experimental protocol is the following: the pressure cell is initially sealed by hand on the bench at a low pressure (typically below $1 \mathrm{GPa}$ ) and all subsequent pressure changes are performed in situ using the membrane. We adjust the pressure at the upper limit of our temperature
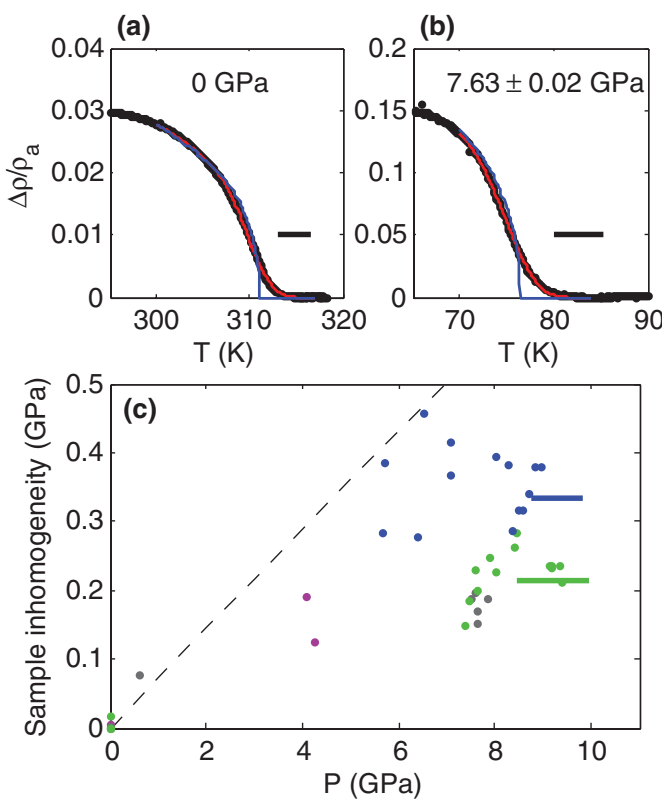

FIG. 4. Pressure inhomogeneity across our sample at low temperature. (a) and (b) Magnetic resistivity (black points) and best-fits (red lines) using Eq. (1) for one sample at both ambient and high pressure; $\delta T_{\mathrm{N}}$ is graphically indicated by the black bars. Also shown (blue lines) is the fit function evaluated with $\delta T_{\mathrm{N}}=0$ and the other three fitting parameters held at their best-fit values. (a) At $P=0 \mathrm{GPa}, T_{\mathrm{N}}=311.35 \pm 0.02 \mathrm{~K}$ and $\delta T_{\mathrm{N}}=3.6 \mathrm{~K}$. (b) At $\mathrm{P}=7.63 \pm 0.02 \mathrm{GPa}, T_{\mathrm{N}}=76.40 \pm 0.04 \mathrm{~K}$ and $\delta T_{\mathrm{N}}=5.2 \mathrm{~K}$. (c) Pressure inhomogeneity. Individual points correspond to fits as in (a) and (b), and different colors indicate different samples; colors are consistent with Fig. 3. The temperature of the data follows the magnetic phase diagram; ${ }^{1}$ e.g., $T<100 \mathrm{~K}$ for $P>6 \mathrm{GPa}$. The bars represent the FWHM of the pressure inhomogeneity from power law fits to $\Delta \rho / \rho_{\mathrm{a}}$ isotherms at $T=5 \mathrm{~K}$ (see text and Ref. 1); the colors indicate different samples and correspond to the individual points. For these bars the vertical position is the pressure inhomogeneity, and the horizontal length is the range of the power law fit. The dashed line is an upper bound on the pressure inhomogeneity determined in an equivalent experimental setup. ${ }^{18}$

sweeps; for the data collected in the quantum critical regime this means that the pressure is increased with the cell at $75 \mathrm{~K}$ or above. Our lowest measurement temperature is limited by the liquid-solid transition of the helium in the membrane line. Helium freezes at 2030 psi at $\mathrm{T}=4.2 \mathrm{~K}$, which is comparable to the membrane pressures used in these experiments. If the cell temperature is lowered through this transition then there is an abrupt change in the membrane pressure and a corresponding change in the sample pressure. This effect often precludes measuring the resistivity $\rho(T)$ at a fixed pressure through the full experimentally accessible temperature range, and for this reason, we limited the measurements to $4.5 \mathrm{~K}$ and above. The cryostat sits in the bore of a room-temperature electromagnet, which provides a \pm 0.5 Tesla field for measuring the Hall coefficient. All electrical measurements were made using an LR700 (Linear Research) ac resistance bridge. In Figs. 3-5, we present data measured from five samples using the experimental techniques described above. Among those five samples, two were measured extensively in the vicinity of the quantum critical pressure near $10 \mathrm{GPa}$. Measurements on all other samples were limited to pressures below $8 \mathrm{GPa}$ by assorted failure modes. No sample was reused after pressurization. 
As with any metal, the residual resistivity $\left(\rho_{0}\right)$ is a good measure of the sample quality. In Fig. 3(a), we plot $\rho_{0}$ measured as a function of pressure for two samples that were studied in fine detail in the vicinity of the SDW quantum critical pressure, $P_{\mathrm{c}}(T \rightarrow 0)=9.71 \pm 0.08 \mathrm{GPa}$ (Ref. 1). Both samples show the same steep drop in $\rho_{0}$ across the quantum phase transition. This drop is the result of the enlargement of the Fermi surface when the spin density wave is eliminated, and the steep curve is the result of the narrow quantum critical regime. For both crystals, the residual resistivity of the high-pressure paramagnetic state is below $0.1 \mu \Omega \mathrm{cm}$, characteristic of a clean metal. In the same plot, we reproduce $\rho_{0}$ reported in Ref. 7. These measurements were performed with a Cr sample of size $(20 \times 20 \times 600) \mu \mathrm{m}^{3}$ in a Bridgman cell using steatite as pressure medium. The reported $\rho_{0}$ at high pressure is very similar to our results. This may result in part from the fact that both experiments used samples derived from $\mathrm{Cr}$ single crystals purchased from Alfa Aesar. ${ }^{30} \mathrm{In}$ contrast to these results with an alcohol pressure medium in a DAC and with steatite in a Bridgman cell, using silicone oil as the pressure medium in a DAC results in a rapid deterioration in sample quality (cf. Fig. 2(e)), with the residual resistivity increasing to more than $0.5 \mu \Omega \mathrm{cm}$ at $5.6 \mathrm{GPa}$.

The relative resistivity ratio (RRR) is another useful quality metric for a metallic sample. After spot welding the contacts, but before loading into the pressure cell, we measured $\mathrm{RRR}_{300}=\rho(300 \mathrm{~K}) / \rho(5 \mathrm{~K})$ in the range $85-100$ for our miniature samples. For our high pressure experiments, we did not record data up to room temperature at all pressures (see above), so we cannot report $\mathrm{RRR}_{300}$ at all pressures. However, as demonstrated in Fig. 3(b), the ratio $\mathrm{RRR}_{120}=\rho(120 \mathrm{~K}) /$ $\rho(5 \mathrm{~K})$ remains relatively constant between ambient pressure and the highest measured pressures, even with the partial gapping of the Fermi surface. For the sample measured to the highest pressure, we find that $\mathrm{RRR}_{120}$ changes from 19.5 at $0.80 \mathrm{GPa}$ to 15.9 at $11.35 \mathrm{GPa}$. In between these limits, $\mathrm{RRR}_{120}$ varies non-monotonically in response to the antiferromagnetic phase diagram.

The residual resistivity and the relative resistivity ratio are not the only available metrics for gauging sample quality and the pressure environment. The effects of pressure inhomogeneity on the sample can also be estimated by measuring response functions that depend on pressure and fitting the data to extract the pressure inhomogeneity across the sample. In particular, we analyze the magnetic or excess resistivity $\Delta \rho(T)=\rho(T)-\rho_{\mathrm{PM}}(T)$, defined as the measured resistivity in the antiferromagnetic phase $(\rho(T))$ minus the hypothetical resistivity in the paramagnetic phase $\left(\rho_{\mathrm{PM}}(T)\right)$. The latter can be modeled by extending a fit to the $T>T_{\mathrm{N}}$ data, or can be taken directly from measurements made at higher pressure; the best procedure depends on the pressure and temperature range under consideration. The temperature-dependence of magnetic resistivity can be fit to a model that accounts for thermal excitation of charge carriers over the SDW energy gap, ${ }^{19,31}$

$$
\frac{\Delta \rho}{\rho}=\left(q^{-1}+1\right)^{-1}\left[1-\int_{-\infty}^{\infty} d \xi \frac{\Delta^{2}}{E^{3}} \frac{1}{e^{\beta E}+1}\right] .
$$

Here $\Delta(T)$ is the SDW exchange energy, $E=\sqrt{\xi^{2}+\Delta^{2}}$, $\beta=\left(k_{B} T\right)^{-1}$, and $q$ is a ratio that depends on the rela- tive density of states on the magnetic and non-magnetic portions of the Fermi surface. The exchange energy $\Delta(T)$ is assumed to evolve in temperature according to the gap equation from the Bardeen-Cooper-Schrieffer (BCS) theory of superconductivity. ${ }^{33}$ Fitting Eq. (1) to data involves three free parameters: the low-temperature value $\Delta(T \rightarrow 0) \equiv \Delta_{0}$, the critical temperature $T_{\mathrm{N}}$, and $q$.

We plot in Figs. 4(a) and 4(b) examples of the measured magnetic resistivity at ambient pressure and at $7.6 \mathrm{GPa}$ for one sample. The functional form described by Eq. (1) contains a singularity at $T_{\mathrm{N}}$ due to the critical form of the BCS gap equation (see the blue lines in Figs. 4(a) and 4(b). In practice, this feature is smeared out by inhomogeneities, including inhomogeneity in the pressure across the sample, and the data are rounded near $T_{\mathrm{N}}$. We account for these inhomogeneities by numerically convoluting Eq. (1) with a Gaussian distribution of $T_{\mathrm{N}}$ with full width of half maximum (FWHM) $\delta T_{\mathrm{N}}$. We assume that both $\Delta_{0}$ and $q$ vary linearly with and in proportion to the variation in $T_{\mathrm{N}}$ such that $\delta q / q$ $=\delta \Delta_{0} / \Delta_{0}=\delta T_{N} / T_{N}$ (for all but the highest pressures the fit results are insensitive to different choices for the effects of pressure inhomogeneity on $\Delta_{0}$ and $q$ ). Our best-fit (red lines in Figs. 4(a) and 4(b) therefore uses four free parameters $\left(T_{\mathrm{N}}, \delta T_{\mathrm{N}}, q, \Delta_{0}\right)$ to model the data. The latter two, $q$ and $\Delta_{0}$, are highly correlated and are poorly constrained unless the $\Delta \rho / \rho$ data extend to sufficiently low temperature so that the function described by Eq. (1) reaches saturation. In contrast, $T_{\mathrm{N}}$ and $\delta T_{\mathrm{N}}$ are well constrained even for a limited range of available data. In Figs. 4(a) and 4(b), we show the best-fit curves as well as the results of evaluating the fit function with the same best fit parameters except for $\delta T_{\mathrm{N}}$, which is set to zero. It is clear from this comparison that $\delta T_{\mathrm{N}}$ is determined primarily by the rounding of the data near the $\Delta \rho / \rho=0$ axis.

Here we focus on $\delta T_{\mathrm{N}}$ and the pressure inhomogeneity experienced by the sample. The pressure inhomogeneity $\delta P=\left(\frac{d T_{N}(P)}{d P}\right)^{-1} \sqrt{\left(\delta T_{N}(P)\right)^{2}-\left(\delta T_{N}(0)\right)^{2}}$ is calculated from $\delta T_{\mathrm{N}}$ using the known magnetic phase diagram $T_{\mathrm{N}}(P){ }^{1} \delta T_{\mathrm{N}}$ at ambient pressure is always in the range $3-6 \mathrm{~K}$ and reflects both the influence of sample imperfections and the small deviation of the Néel transition from the mean-field limit. ${ }^{32}$ The compiled results for multiple crystals (Fig. 4(c)) demonstrate that the pressure inhomogeneity is always below 5\% in the range 8-10 GPa. In Fig. 4(c), we also plot an upper bound (dashed line) for the pressure inhomogeneity expected in this type of experimental assembly for a $(200 \times 200) \mu \mathrm{m}^{2}$ square sample. This upper bound was estimated from a systematic study of the pressure variation within a pressure chamber slightly smaller than that used in this work, and was set to contain the full range of experimental observations. ${ }^{18}$ We are satisfied that the values measured here, which are the FWHM of a Gaussian distribution function, fall well below this upper bound. We also note that the pressure inhomogeneity described here is larger than that seen in x-ray diffraction experiments that used a sample $1 / 6^{\text {th }}$ the size. ${ }^{18}$ This comparison emphasizes the importance of minimizing the volume occupied by the sample within a given pressure chamber. For future work, it is conceivable that laser dicing could be used to produce a clover-shaped sample, ${ }^{26}$ 
which would reduce the effective sample area and the pressure inhomogeneity.

For $P>9.5 \mathrm{GPa}$, it becomes difficult to fit Eq. (1) to the measured data for two reasons. First, our measurements do not extend below $T=4.5 \mathrm{~K}$ and the measured $\Delta \rho / \rho$ curves do not constrain well the fit parameters. Second, in the critical regime the assumption that small spreads in pressure correspond to small spreads in the fit parameters breaks down due to the singular behavior of $T_{\mathrm{N}}(P)$ at $P_{\mathrm{c}}(T \rightarrow 0)$. Therefore, in the critical regime we instead use data measured during successive temperature sweeps at closely spaced pressures to reconstruct $\Delta \rho / \rho$ as a function of pressure at fixed temperatures. We fit isotherms to a power law $\Delta \rho / \rho=a\left(P_{c}(T)-P\right)^{\beta}$, convolved with a Gaussian pressure distribution. The fit parameters $P_{\mathrm{c}}(T)$ and $\beta$ describe the quantum critical behavior, ${ }^{1}$ and the full width of the pressure distribution depends on the condition of the sample in the pressure chamber. The pressure distribution estimated in this way for two samples measured in fine detail in the quantum critical regime is shown in Fig. 4(c), and is consistent with the values estimated from fits of Eq. (1) to data at lower pressure. We note that the results reported here are little affected by the assumed functional form of the pressure inhomogeneity. For example, fitting the data with a triangle distribution function results in a FWHM that is within $1 \%$ of the Gaussian FWHM. Using the square distribution results in a full width that is within $20 \%$ of the Gaussian FWHM.

We show in Fig. 5 the results of fitting the resistivity $\rho(T, P)$ to the Bloch-Gruneisen formula, which describes the temperature dependence of the resistivity of a metal assuming a single conduction band and a conductivity limited by phonon scattering. ${ }^{34}$ This formula provides a good fit to the data except in the vicinity of $T_{\mathrm{N}}$ or at low temperature (approximately $T<20 \mathrm{~K}$ ), where the power law deviates from $T^{5} .{ }^{1}$ The fitting is performed with the help of an analytic approximation to the Bloch-Gruneisen function. ${ }^{35}$ In Fig. 5(a), we show the resistivity and fit at ambient pressure. The fit range is $30-200 \mathrm{~K}$, well within the antiferromagnetic phase $\left(T_{\mathrm{N}}(P=0)=311 \mathrm{~K}\right)$, and the extracted Debye temperature $(\Theta)$ is $535 \pm 1$ K. In Fig. 5(d), we show the resistivity and fit at $11.35 \pm 0.01 \mathrm{GPa}$. This is well above the quantum critical pressure, so the data are entirely in the non-magnetic phase; here also $\Theta$ $=544 \pm 1 \mathrm{~K}$. Using data compiled from 7 crystals (Fig. 5(e)), we find that the Debye temperature is essentially independent of pressure in the range 0-11.35 GPa. In Fig. 5(e), the fit results are separated into fits in the magnetic and non-magnetic phases. These two groups are cleanly separated into low- and high-pressure regimes, respectively, by the particulars of the experiment. The temperature range available for fitting in the magnetic phase is limited by the transition temperature $T_{\mathrm{N}}(P)$, and the available range in the non-magnetic phase is limited by the experimental protocol. We believe that the slight decrease in $\Theta$ between 2 and $6 \mathrm{GPa}$ is an artifact resulting from the limited temperature range available for fitting in the magnetic phase, a systematic issue which gets worse as $T_{\mathrm{N}}$ is suppressed with pressure. This is quantified by the errorbars in Fig. 5(e), which show the systematic variations in $\Theta_{D}$ that result from applying different fit constraints. Given this uncer-
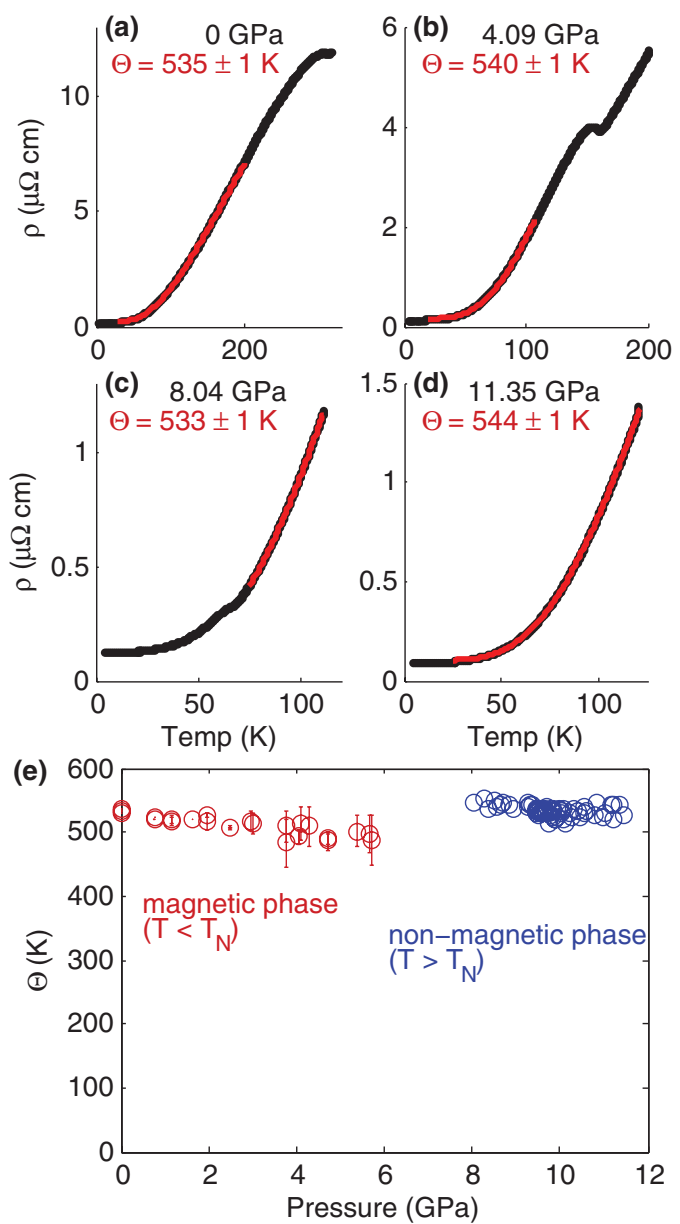

FIG. 5. Fitting the resistivity $\rho(T, P)$ to the Bloch-Gruneisen equation. In (a)-(d), the data are black and the best-fit curve is red. (a) Data and fit at ambient pressure, giving Debye temperature $\Theta=535 \pm 1 \mathrm{~K}$. Fit range $30-200 \mathrm{~K}$ is in the magnetic phase. (b) Data and fit at $4.09 \mathrm{GPa}$. The fit range $20-107 \mathrm{~K}$ is in the magnetic phase and $\Theta=540 \pm 1 \mathrm{~K}$. (c) Data and fit at $8.04 \mathrm{GPa}$. The fit range $75-110 \mathrm{~K}$ is in the non-magnetic phase and $\Theta$ $=533 \pm 1 \mathrm{~K}$. (d) Data and fit at $11.35 \mathrm{GPa}$, above the quantum critical pressure (data are entirely in the non-magnetic phase). The fit range is $25-120 \mathrm{~K}$ and $\Theta=544 \pm 1 \mathrm{~K}$. (e) Fit results $\Theta(P)$ for seven Cr crystals measured between 0 and $11.35 \mathrm{GPa}$. The results are separated into fits in the magnetic and non-magnetic phases. Errorbars for results in the magnetic phase are shown; errorbars in the non-magnetic phase are all smaller than the symbol size.

tainty, we cannot firmly rule out that the crystal lattice softens in the vicinity of $T_{\mathrm{N}}$. However, our results strongly support the conclusion that $\Theta_{\mathrm{D}}$ is equal in the high pressure paramagnetic phase (above $10 \mathrm{GPa}$ ) and the low pressure antiferromagnetic phase (approximately below $2 \mathrm{GPa}$ ).

We are not aware of any published reports of $\Theta$ determined for chromium by analyzing the electrical resistivity, nor at high pressure. However, our values for $\Theta$ compare well to reports in the literature of measurements made by other techniques at ambient pressure. X-ray measurements on bulk single crystals find $\Theta=545 \mathrm{~K}$ at room temperature, ${ }^{36}$ specific heat measurements on bulk polycrystals find $\Theta=580$ for $T>14 \mathrm{~K},{ }^{37}$ and specific heat measurements on thin films find $\Theta=405-500 \mathrm{~K}$, with lower values for more disordered films. ${ }^{38}$ 


\section{CONCLUSION}

We have described and demonstrated a technique for making four-probe electrical transport measurements in a van der Pauw configuration at high pressure and low temperature in a diamond anvil cell using a quasi-hydrostatic liquid pressure medium. The liquid pressure medium preserves sample quality for $P>11 \mathrm{GPa}$, opening the door to high-resolution studies of phase transitions and critical phenomena. Our technique uses a composite gasket made of stainless steel and an $\mathrm{Al}_{2} \mathrm{O}_{3}$-filled epoxy to electrically isolate the leads from the gasket and from each other. Our approach draws from a number of techniques published previously in this journal, most notably Refs. 9 and 11, to which we add the capability of using an alcohol pressure medium. Using our technique, we have been able to measure the antiferromagnetic phase diagram and quantum critical scaling exponents in elemental $\mathrm{Cr}$ through measurements of the resistivity and Hall coefficient. ${ }^{1}$ We have evaluated quantitatively the sample quality at high pressure by investigating the effects of pressure inhomogeneity and accumulated sample damage on the residual resistivity and the antiferromagnetic transition. In addition, the Debye temperature of $\mathrm{Cr}$ was determined for pressures up to 11.4 GPa using the Bloch-Gruneisen formula and was found to be independent of pressure.

\section{ACKNOWLEDGMENTS}

The work at the University of Chicago was supported by National Science Foundation Grant No. DMR-1206519. The work at the Advanced Photon Source of the Argonne National Laboratory was supported by the U.S. DOE-BES under Contract No. DE-AC02-06CH11357.

${ }^{1}$ R. Jaramillo, Y. Feng, J. Wang, and T. F. Rosenbaum, Proc. Natl. Acad. Sci. U.S.A. 107, 13631 (2010).

${ }^{2}$ H. Taniguchi et al., J. Phys. Soc. Jpn. 72, 468 (2003).

${ }^{3}$ L. G. Khvostantsev, V. N. Slesarev, and V. V. Brazhkin, High Press. Res. 24, 371 (2004).

${ }^{4}$ T. Nakanishi, N. Takeshita, and N. Môri, Rev. Sci. Instrum. 73, 1828 (2002).
${ }^{5}$ E. Colombier and D. Braithwaite, Rev. Sci. Instrum. 78, 093903 (2007).

${ }^{6}$ A.-S. Rüetschi and D. Jaccard, Rev. Sci. Instrum. 78, 123901 (2007).

${ }^{7}$ P. Pedrazzini and D. Jaccard, Physica B 403, 1222 (2008).

${ }^{8}$ R. L. Reichlin, Rev. Sci. Instrum. 54, 1674 (1983).

${ }^{9}$ S. W. Tozer and H. E. King, Rev. Sci. Instrum. 56, 260 (1985).

${ }^{10}$ J. Gonzalez, J. M. Besson, and G. Weill, Rev. Sci. Instrum. 57, 106 (1986).

${ }^{11}$ D. Erskine, P. Y. Yu, and G. Martinez, Rev. Sci. Instrum. 58, 406 (1987).

${ }^{12}$ T. Adachi, H. Tanaka, H. Kobayashi, and T. Miyazaki, Rev. Sci. Instrum. 72, 2358 (2001).

${ }^{13}$ M. I. Eremets, V. V. Struzhkin, H.-k. Mao, and R. J. Hemley, Science 293, 272 (2001).

${ }^{14}$ K. Shimizu, T. Kimura, S. Furomoto, K. Takeda, K. Kontani, Y. Onuki, and K. Amaya, Nature 412, 6844 (2001).

${ }^{15}$ S. A. Boye, D. Rosén, P. Lazor, and I. Katardjiev, Rev. Sci. Instrum. 75, 5010 (2004)

${ }^{16}$ A. B. Garg, V. Vijayakumar, and B. K. Godwal, Rev. Sci. Instrum. 75, 2475 (2004).

${ }^{17}$ S. Yomo and S. W. Tozer, J. Phys.: Conf. Ser. 215, 012181 (2010).

${ }^{18}$ Y. Feng, R. Jaramillo, J. Wang, Y. Ren, and T. F. Rosenbaum, Rev. Sci. Instrum. 81, 041301 (2010).

${ }^{19}$ D. B. McWhan and T. M. Rice, Phys. Rev. Lett. 19, 846 (1967).

${ }^{20}$ K. Shimizu, H. Ishikawa, D. Takao, T. Yagi, and K. Amaya, Nature 419, 597 (2002).

${ }^{21}$ M. Debessai, T. Matsuoka, J. J. Hamlin, J. S. Schilling, and K. Shimizu, Phys. Rev. Lett. 102, 197002 (2009).

${ }^{22}$ J. van Straaten and I. F. Silvera, Rev. Sci. Instrum. 58, 994 (1987).

${ }^{23}$ H. Hemmes et al., Rev. Sci. Instrum. 60, 474 (1989).

${ }^{24}$ D. D. Jackson et al., Phys. Rev. B 74, 174401 (2006).

${ }^{25}$ D. J. Dunstan and I. L. Spain, J. Phys. E: Sci. Instrum. 22, 913 (1989).

${ }^{26}$ L. J. van der Pauw, Philips Res. Rep. 13, 1 (1958).

${ }^{27}$ T. Hiraoka, Rev. Sci. Instrum. 69, 2808 (1998).

${ }^{28}$ I. R. Walker and C. J. Moss, Rev. Sci. Instrum. 69, 2747 (1998).

${ }^{29}$ Y. Feng, M. S. Somayazulu, R. Jaramillo, T. F. Rosenbaum, E. D. Isaacs, J. Hu, and H.-k. Mao, Rev. Sci. Instrum. 76, 063913 (2005).

${ }^{30}$ No longer available at the time of writing.

${ }^{31}$ D. Jérome, T. M. Rice, and W. Kohn, Phys. Rev. 158, 462 (1967).

${ }^{32}$ R. Jaramillo, Y. Feng, J. C. Lang, Z. Islam, G. Srajer, H. M. Ronnow, P. B. Littlewood, and T. F. Rosenbaum, Phys. Rev. B 77, 184418 (2008).

${ }^{33}$ A. W. Overhauser, Phys. Rev. 128, 1437 (1962).

${ }^{34} \mathrm{~J}$. M. Ziman, Electrons and Phonons (Oxford University Press, London, 1960), pp. 357-367.

${ }^{35}$ M. Deutsch, J. Phys. A 20, L811 (1987).

${ }^{36}$ R. H. Wilson, E. F. Skelton, and J. L. Katz, Acta Crystallogr. 21, 635 (1966).

${ }^{37}$ F. Heininger, E. Bucher, and J. Muller, Z. Phys. B: Condens. Matter 5, 243 (1966); K. Clusius and P. Franzosini, Z. Naturforsch Pt A 17, 522 (1962).

${ }^{38}$ D. W. Cooke, Z. Boekelheide, D. R. Queen, and F. Hellman, J. Appl. Phys. 105, 07C314 (2009). 